\title{
EL USO DE CRIPTOMONEDAS EN CROWDFUNDING Y EN OTRAS ESTRATEGIAS DE NEGOCIO PARA PROMOVER EL CRECIMIENTO EMPRESARIAL INTERNACIONALMENTE
}

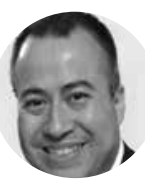

\author{
PEDRO R. BERDEJO \\ - Profesor de Marketing \\ Dirección de Administración y Marketing \\ Facultad de Negocios, UPC
}

\section{RESUMEN}

Esta investigación analiza algunas de las principales ventajas y oportunidades de mejora que plantea el empleo de las criptomonedas en el crowdfunding ${ }^{1}$ y en otras estrategias de negocio en una empresa o holding para promover su crecimiento internacionalmente. Se analizan las ventajas del uso de las criptomonedas para financiar proyectos mediante las initial coin offerings, tanto para los emisores, como para los inversionistas; así como las oportunidades de mejora necesarias regulatoriamente, de modo que se proteja a estos últimos de estafas y se consolide la aceptación plena de esta modalidad colaborativa de financiamiento sin intermediarios en la comunidad empresarial globalmente.

Palabras Claves: criptomonedas, tokens, crowdfunding, initial coin offering, financiamiento virtual, sistemas contables distribuidos, blockchain, tangle.

\section{ABSTRACT}

This paper analyzes some of the main advantages and opportunities for improvement posed by the use of cryptocurrencies in crowdfunding and other business strategies in a firm or company holding in order to promote its growth internationally. It places specific attention to the advantages of using cryptocurrencies to finance projects through initial coin offerings, for both issuers and investors. Attention is also placed on the regulatory improvement opportunities needed for both protecting them, but especially the latter, from scams, and consolidating full acceptance of this financing option in the international business community.

Keywords: cryptocurrencies, tokens, crowdfunding, initial coin offering, virtual funding, distributed accounting systems, blockchain, tangle.

\section{INTRODUCCIÓN}

Tal como las define Satoshi Nakamoto, creador del bitcoin (Nakamoto, 2008), una criptomoneda es un sistema de pago basado en prueba criptográfica en vez que en la confianza en una tercera parte. La prueba criptográfica consiste de una cadena de firmas digitales de los sucesivos propietarios de cada unidad de moneda. Cada propietario transfiere la moneda al siguiente firmando digitalmente con una clave criptográfica de tamaño fijo llamada "hash" que contiene la clave de la transacción previa y la clave pública de identificación del siguiente propietario. El beneficiario puede verificar las firmas para verificar la cadena de propiedad.

Como indican De Albuquerque y De Castro Callado (2015), el término de criptomoneda fue usado por primera vez por Wei Dai en 1998 en un artículo publicado en un grupo de Internet conocido como cypherpunks ${ }^{2}$ donde brinda una solución al problema de pagos descentralizados y electrónicos.

La primera criptomoneda que se creó exitosamente fue el bitcoin. Ésta apareció entre 2008 y 2009 teniendo como contexto la crisis financiera internacional y la recesión global ocasionada por el derrumbe de las hipotecas subprime en Estados Unidos. Sin embargo, no fue sino hasta mayo de 2010, cuando bitcoin comenzó a adquirir valor, al comprar un usuario dos pizzas por dos mil bitcoins. Desde la creación de bitcoin, se han creado una numerosa variedad de criptomonedas con diferentes características y protocolos como litecoin, ether (ethereum), ripple, dogecoin, iota. bitcoin, a fines de 2017 alcanzó un valor de mas de 18 mil dólares al lanzarse al mercado de futuros en Wall Street basados en esta criptomoneda ${ }^{3}$. Actualmente, el valor del bitcoin tiende a estabilizarse entre seis y siete mil dólares, luego de una corrección de alrededor del 70\% a la baja del precio máximo alcanzado en diciembre de 2017.

La tecnología que permitió la creación del bitcoin se denomina blockchain (cadena de bloques). Blockchain y otras tecnologías similares, como la plataforma "Tangle"4,

2. Ver http://www.weidai.com/bmoney.txt

3. Ver https://www.criptonoticias.com/mercado-valores/primeros futuros-bitcoin-cme-cierran-18500/

4. Ver https://iota.org/ 
basada en tecnología $\mathrm{DAG}^{5}$, que emplea la criptomoneda iota, son lo que se conoce como sistemas de contabilidad digital distribuida o sistemas DLT, por sus siglas en inglés (Distribuited Ledger Technology). Estos sistemas permiten registrar de forma irreversible transacciones de un modo que es prácticamente imposible "hackear". Esta característica hace viable su uso para registrar de forma irreversible la transferencia de propiedad de un sin número de activos digitales (e.g., contratos, patentes, música y otras obras no físicas). A partir de las plataformas DTL, como Ethereum ${ }^{6}$, se puede generar también tokens o criptomonedas para fines específicos u otro tipo de activos digitales, como "contratos inteligentes" de una forma relativamente simple y rápida.

Por definición, el uso principal de una criptomoneda es servir de unidad de cambio. Sin embargo, en los últimos años también se están empleando como instrumentos de título valor, siendo emitidas por la participación como inversionista en un proyecto entre otros usos empresariales posibles. De hecho, en los últimos años, desde su primer uso bajo esta modalidad en 2013 por Mastercoin (Chohan, 2017), miles de millones de dólares han sido recaudados a traves de criptomonedas bajo esta modalidad de crowdfunding denominada Initial Coin Offering (ICO). Por otro lado, tambien se han creado fondos de inversión en criptomonedas, como Fisco Ltd. en Japón, quien en agosto del 2017 lanzó el primer bono corporativo en bitcoins para una de las empresas de su grupo económico ${ }^{7}$, dentro de un contexto de mejoras en la ley y reglamentaciones que reconocen al bitcoin como un instrumento de pago legal ${ }^{8}$.
Dado este reciente uso de las criptomonedas como estrategia empresarial, la pregunta que buscaremos responder en esta investigación es la siguiente: ¿qué ventajas potenciales y oportunidades de mejora plantea el uso de las criptomonedas en crowdfunding y como herramienta para estrategias de crecimiento empresarial? Lo que motiva el desarrollo de esta investigación es comprobar el potencial de las criptomonedas para democratizar el financiamiento para las pequeñas empresas y potenciar su desarrollo comercial internacionalmente en el contexto globalizado actual.

\section{MÉTODO DE INVESTIGACIÓN Y DESARROLLO}

\subsection{METODOLOGÍA}

La población sujeta de investigación es la de las empresas que han empleado criptomonedas para obtener financiamiento por crowdfunding. El método empleado para esta investigación será el análisis de casos documentados de las ICOs en los sitios web de estos proyectos y la revisión de investigaciones científicas de revistas arbitradas sobre los avances en la regulación de ICOs y criptomonedas.

\subsection{ALGUNAS ESTADÍSTICAS DE CONTEXTO:}

Durante 2017 se recaudaron más de 3,800 MM USD a través de 210 ICO solo en los Estados Unidos (ver Gráfico 1) y se proyecta que, para el 2018 , este monto supere los 20,000 MM USD con más del doble de ICO?.

\section{Gráfico 1: Ofertas iniciales de Criptomonedas (2017)}

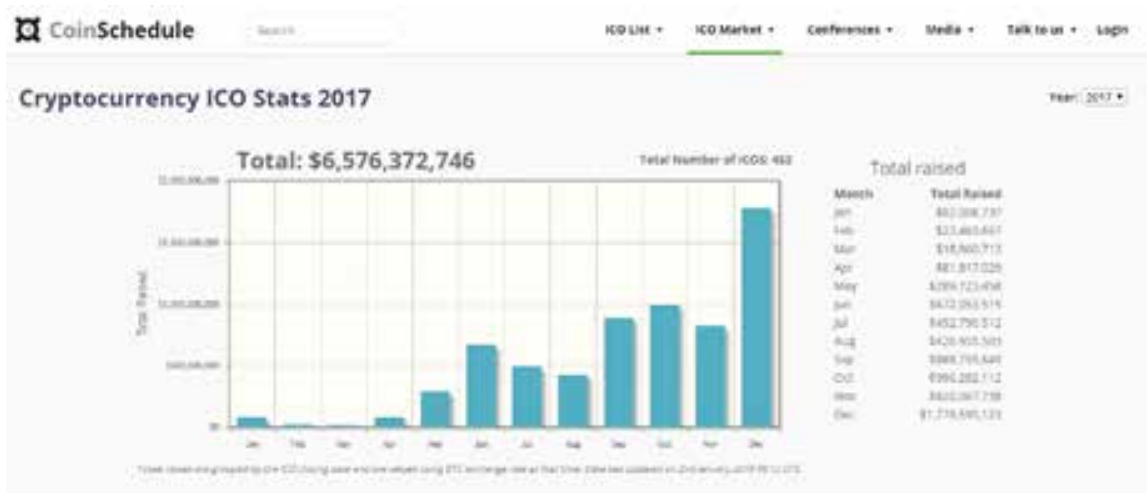

Fuente: CoinSchedule https://www.coinschedule.com/stats.html?year=2017

6 Para mayor información, ver https://www.ethereum.org/

7. Ver https://www.crypto-economy.net/es-PE/japon-emite-suprimer-bono-bitcoin/

8. Ver https://www.criptonoticias.com/adopcion/japon-legalizabitcoin-metodo-pago/ 
En este feroz crecimiento no han faltado estafas en un entorno donde los organismos reguladores financieros de los gobiernos continúan en un trabajo arduo para crear marcos legales para reducir la incertidumbre de estos mercados.

\subsection{ANÁLISIS DE CASOS:}

A continuación, analizamos el uso de criptomonedas en 3 casos innovadores: a) Telegram; b) Kodak; c) Crypterium.

\section{Caso 1: Telegram y su moneda "gram".}

Telegram es un servicio de mensajería similar a "WhatsApp" y a "Víber" que se basa en una plataforma de código abierto. Es gratuito y limpio de publicidad. Fue iniciado por el empresario ruso Pavel Durov en agosto de 2013. Es uno de los servicios de mensajería más populares y más descargados: a 2018 cuenta con más de 200 millones de usuarios activos). Actualmente la compañía busca desarrollar un proyecto al que denomina Telegram Open Network (TON), una blockchain multidimensional de última generación. Para recaudar capital para el proyecto optó por la emisión de gram, su criptomoneda, vía ICO. La empresa realizó dos ofertas ICO levantando 1,700 MM USD, la primera a mediados de febrero de 2018 y la última a fines de marzo de dicho año, cada una por 850 MM USD ${ }^{10}$. Según una fuente de The Wall Street Journal ${ }^{11}$, Telegram decidió cancelar la ICO desde mayo pues superó ampliamente los 1,200 MM USD planificados con un grupo de menos de 200 inversionistas. Otras fuentes creen que el cierre también podría deberse a las regulaciones cada vez más estrictas que la Securities and Exchange Comission ${ }^{12}$ (SEC) la Commodity Futures Trading Commission (CFTC), están proponiendo desde inicios de año ${ }^{13}$. Sin embargo, antes de que se retirara la página web oficial ${ }^{14}$ pudimos observar en su diseño los componentes para generar interés y atraer a los inversionistas (ver imagen en la Figura 1). Hay un video introductorio al proyecto. Luego en el menú superior están los accesos al contenido informativo de la página web ("What is Gram", "Features", "Our Team", "Roadmap", "White Paper") que hace una explicación del proyecto: En What is Gram brindan información sobre que es la criptomoneda gram y el concepto del proyecto TON (Telegram Open Network) para el cual están solicitando inversionistas. En Features resumen las características innovadoras de TON para transferir información de activos digitales encriptados, de manera rápida y flexible gracias a su arquitectura escalable y multidimensional. La explicación es suficiente para el público objetivo al que se dirige: inversionistas que conocen de tecnología digital. En Our

10. Ver http://www.eleconomista.es/mercados-cotizaciones/ noticias/9043193/04/18/Telegram-recauda-1378-millones-yprotagoniza-la-mayor-ICO-de-la-historia.html

11. Ver https://www.wsj.com/articles/telegram-messaging-appscraps-plans-for-public-coin-offering-1525281933?ns=prod/ accounts-wsj

12. Agencia regulatoria de títulos valores de los EE.UU

13. Ver https://hipertextual.com/2018/05/telegram-echa-atrassu-ico

14. Fuente: Página oficial de la ICO Telegram: https://lco-telegram. $\mathrm{com} /$
Team presentan al equipo que está al frente del proyecto. El Roadmap es el cronograma del proyecto. En el White Paper se muestra una explicación técnica del proyecto y de los pasos de la emisión de la criptomoneda. Esta es una versión ligera del Technical White Paper de 132 páginas publicado en diciembre de 2017, por el Dr. Nicolai Durov, hermano de Pavel Durov y co-fundador de Telegram ${ }^{15}$.

\section{Figura 1}

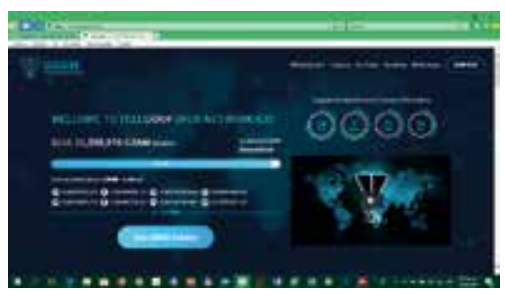

Fuente: https://ico-telegram.com/

En el White Paper, se explican los beneficios que se ofrecen a los tenedores de su criptomoneda gram, entre ellas, resaltamos:

1. Comprar con la criptomoneda activos digitales o físicos que se vendan por empresas en la plataforma TON

2. Servir de depósito para desempeñar la función de "validador de transacciones" en el ecosistema TON y obtener más grams por desempeñar esa función.

3. Tener poder de voto para apoyar u oponerse a cambios en los parámetros de la plataforma TON.

4. Comprar nombres de dominio, hosting y espacio de almacenamiento seguro y descentralizado.

Para la primera ICO, que se llevó a cabo en febrero, se restringió la oferta a inversionistas que compren un mínimo de 1 millón de dólares en criptomoneda y que cumplan con estar acreditados como inversores en la SEC ${ }^{16}$. La venta de los 850 millones de dólares en grams se hizo a 81 inversores acreditados. En la última ICO se hizo la venta de los otros 850 millones de dólares a 94 inversores acreditados.

Según el White Paper del proyecto, la masa monetaria que se emitirá será de 5,000 millones de grams en total. De este total, 4\% (200 millones) se reservan para el equipo de desarrollo en un período de adjudicación de 4 años. Durante la etapa de desarrollo, hasta fines del 2018, al menos el 52\% de toda la oferta será retenida para proteger a la naciente criptomoneda del comercio especulativo y para mantener la flexibilidad en las primeras etapas de evolución del sistema. El restante 44\% (2,200 millones) se pone a la venta. El primer token se vendió a 0.1 USD y cada uno de los sucesivos a un precio de una mil millonésima más que el anterior. Esta estructura de emisión debe permitir que el mercado defina el precio justo y el volumen para la venta del token. Es posible realizar una venta a

15. Ver https://www.docdroid.net/JtdY7Jg/ton.pdf

16. SEC: United States Securities Exchanges Commission 
granel de grams a un grupo de inversores institucionales por debajo del precio promedio por token según sea la fecha y el volumen de transacción.

Tanto la primera, como la segunda ICO, se informaron de forma posterior a la SEC a través de un aviso, Form D, en virtud de la Exención Federal 506(c) de los Estados Unidos, que establece que "una empresa puede, en general, solicitar y anunciar la oferta y aun así considerarse que cumple con los requisitos de la exención", siempre y cuando se cumplan ciertas condiciones. En primer lugar, todos los inversores en la oferta deben estar acreditados $\mathrm{y}$, en segundo lugar, el emisor debe tomar medidas razonables para verificar dicha acreditación.

\section{Caso 2: Kodak y su moneda "KodakCoin"}

Tras varios años de mal desempeño, Eastman Kodak anunció en enero de 2012 la reorganización de su negocio y se acogió al Capítulo 11 de la Ley Federal de Quiebras de los EE.UU. entrando en concurso de acreedores ${ }^{17}$. La empresa ha pasado por un proceso de reestructuración y parece resurgir con su nuevo CEO, Jeff Clarke, conocido ejecutivo que ha sido director en empresas como HP, Compuware Corporation y Orbitz, quien fuera elegido para el puesto en marzo de 2014. A partir de 2015, hizo algunos lanzamientos, como su primer teléfono inteligente.

Actualmente se encuentra desarrollando, en alianza con Wenn Digital Partner, el proyecto KodakOne, que es una plataforma digital cifrada y que utilizará el blockchain para la gestión de transacciones. Esta plataforma permitirá a los fotógrafos registrar sus obras (nuevas o antiguas), licenciarlas y recibir pagos por la venta de sus trabajos a través de su propia criptomoneda KodakCoin. Para proteger las imágenes registradas por fotógrafos, la plataforma monitorizará y rastreará la web para detectar el uso indebido de las mismas y se encargará de los procesos legales correspondientes ${ }^{18}$. La misión que pretende cumplir KodakOne, tal y como se muestra en la página web icotokennews.com, es la de crear una comunidad del mundo de la fotografía y de las cadenas de suministros, para fotógrafos de todos los niveles y compradores de imágenes, y que descanse en su propio token. Para financiar el proyecto, se ha anunciado la realización de una ICO, que, como indica su comunicado del 8 de febrero de 2018, está en etapa de rondas de preventa, para lo que se están tomando las medidas necesarias que garanticen que la ICO de KodakCoin cumpla con todas las garantías legales aplicables. Aquí, un primer paso es garantizar que todos los inversores cumplan con los requisitos de calificación de las jurisdicciones aplicables en donde se realizarán las ofertas y ventas. Según lo informado en Business Wire ${ }^{19}$, estas se realizarían en Estados Unidos, Canadá y Reino Unido.

17. Ver https://cincodias.elpais.com/cincodias/2012/01/20/ empresas/1327070387_850215.html

18. Ver https://www.kodak.com/kodakone/default. $h t m ? C I D=g o \& i d h b x=k o d a k o n e$

19. Ver https://www.businesswire.com/news/ home/20180109006183/en/KODAK-WENN-Digital-PartnerLaunch-Major-Blockchain

\section{Caso 3: Crypterium}

Crypterium es un banco que opera con criptomonedas y busca ampliar la cantidad de lugares donde se pueda pagar con estas. Los servicios que ofrece son: i) pagos instantáneos internacionales en moneda tradicional o criptodivisas; ii) emisión de tarjetas virtuales de American Express, Visa, MasterCard, Union Pay; iii) integración con billeteras digitales o servicios de pagos de terceros (e.g., Paypal, Gyft, WeChat, Alipay, PayTM); iv) programas de fidelidad y reembolsos por cada transacción; v) préstamos instantáneos y líneas de crédito multidivisa; vi) pagos sin contacto (e.g., ApplePay, SamsungPay, AndroidPay, QR code); vii) equipo asistencia multilingüe $24 / 7 .^{20}$

Actualmente Crypterium desea financiar un proyecto para desarrollar su propia plataforma de pagos de criptobanca. Según se explica en el White Paper del proyecto ${ }^{21}$, para esta plataforma empleará su propia criptomoneda "crypterium" (CRPT), que habilitará el proceso de transacciones. No se puede realizar ningún pago sin CRPT, su objetivo principal, regulado por contrato inteligente, es estimular a los tenedores de billetes a utilizar crypterium en su vida diaria y atraer nuevos usuarios a Crypterium. Para entender una de las modalidades de aplicación, el pago punto a punto de un precio en divisa convencional con criptomonedas, veamos el siguiente ejemplo: $\mathrm{Si}$ alguien que es cliente de Crypterium, y por ello posee CRPT tokens, quiere usar los bitcoins que tiene en su monedero electrónico, "Crypterium Wallet", para comprar una taza de café que cuesta 10 dólares, solo debe acercar su smartphone al terminal NFC ${ }^{22}$ del vendedor. El servicio de Crypterium en este caso es cambiar instantáneamente a 10 dólares los bitcoins del monedero del cliente. Para pagar este servicio, se hará un recargo del 0.5\% del precio (0.05 dólares) que será pagado en tokens CRPT de la cuenta del cliente, redimiendo así la inversión en tokens CRPT que el cliente compró con anticipación. Actualmente, si una persona que posee bitcoins, en un monedero electrónico cualquiera, desea comprar esa misma taza de café, debe primero cambiarlos en una casa de cambio electrónica, lo que le tomará en el mejor de los casos 20 minutos (por la velocidad de verificación de la blockchain de bitcoin) y luego debe ordenar a la casa de cambio la transferencia de sus fondos en dólares a su cuenta de banco, lo cual puede demorar tres días (si la casa de cambio virtual está en el mismo país). Sólo en ese momento tendrá los dólares en su cuenta para pagar con su tarjeta de débito convencional.

Para habilitar a los clientes al uso de este servicio y otros más de su plataforma en proyecto, Crypterium vendió $210 \mathrm{MM}$ de tokens CRPT a un valor inicial de mercado de 0.0001 bitcoins (BTC) por CRPT. La ICO de Crypterium (CRPT), se realizó del 31 de octubre de 2017 al 14 de enero

\section{Ver https://crypterium.io/spa/index.html\#ca}

21. Ver https://crypterium.io/wp/index, 1.3. Ico Overview, p.6

22. La tecnología NFC, Near Field Communication, está basada en un chip que nos permite comunicar nuestros terminales de forma inalámbrica y por proximidad. Se ha generalizado en nuestros teléfonos sobre todo a partir de 2013, pero no todos lo llevan. Fuente: https://www.androidpit.es/smartphones-tabletssmartwatches-nfc 
de 2018, recaudando 47 MM USD. Según reveló en una entrevista ${ }^{23}$ Austin Kimm, uno de los fundadores, la venta se hizo a 72,000 inversionistas de más de 150 países (un promedio de 650 USD por persona), siendo en este sentido un éxito también por su difusión y acogida. Como se describe en este caso, ese dinero recaudado es más bien un prepago por servicios futuros, que funciona a la vez como financiamiento del proyecto y como estrategia de fidelización y de ampliación de la base de usuarios futuros de Crypterium. No brinda propiedad alguna sobre la compañía ni da mucho menos derecho a voto sobre las decisiones de gestión de la plataforma. Esta inversión se redime con el uso de los servicios en la futura plataforma. Para hacer más atractivo el token CRPT a los compradores, en el White Paper se explica que se premiará con rebates a los clientes-tenedores de tokens a través de un programa de lealtad. Asimismo, el costo de uso de la plataforma por los comercios será de entre 0.5\% a 1\% en vez del 5\% a 6\% de los sistemas Visa, Mastercard y American Express. ${ }^{24}$

\section{REVISIÓN DE HECHOS Y LITERATURA CIENTÍFICA SOBRE EL ESTADO DEL ARTE DE LAS REGULACIONES DE CRIPTOMONEDAS E ICOS}

El inicio del uso de las criptomonedas se da a partir del lanzamiento de bitcoin en 2009. Sin embargo, recién empieza a despegar a partir de mediados de 2010 cuando empiezan a aparecer las primeras o casas de cambio virtuales, en las cuales las personas podían comprar con dólares, euros, libras, etc. los bitcoins y otras criptomonedas de constitución posterior

Actualmente según CoinMarketCap ${ }^{25}$, a la fecha existen cerca de 2,000 criptomonedas cotizando en más de 14,000 mercados virtuales, de las cuales 893 son criptomonedas propiamente dichas, y alrededor de 1,100 son tokens, es decir, monedas de empresas u ecosistemas cerrados emitidos sobre una plataforma base donde se programó el "contrato inteligente" que les permite operar. Estos últimos en buena cuenta dependen de la criptomoneda de la plataforma base.

Por otro lado, las ICOs tienen una historia más reciente. Según la literatura científica, una Initial Coin Offering o ICO es un nuevo y controversial medio de crowdfunding empleando una criptomoneda. Es usada generalmente por una startup para recaudar capital evitando costos de intermediación financiera y regulatorios (Chohan, 2017). También es empleada por empresas ya consolidadas, no solo por startups, ejemplo de ello es Kodak, con la ICO KodakCoin, y la ICO de Home Meal (Mealtoken), la propietaria de la cadena de comida española Nostrum que busca recaudar 50 MM USD para financiar el crecimiento de su cadena de franquicias en un nivel internacional ${ }^{26}$.

23. Ver https://es.dobrebitcoin.com/six-questions-for-crypteriumsumming-up-successful-ico-13508

\section{Ver https://crypterium.io/}

25. CoinMarketCap @ es una página web que lidera la información sobre los mercados de intercambio de criptodivisas.Ver https:// coinmarketcap.com/

26. Ver https://www.bolsasymercados.es/mab/documentos/ HechosRelev/2017/12/05049_HRelev_20171227.pdf
En el caso de Home Meal, la ICO la tendrá que estructurar en Suiza porque ese tipo de operaciones aún no está regulado en España. Sin embargo, según declaraciones del presidente de la Comisión Nacional del Mercado de Valores de España (CNMV), Sebastián Abella, el organismo colaborará con Home Meal para comunicar la ICO: "Hemos hablado con ellos y nuestro deseo sería colaborar en que esa operación se hiciera en España con un folleto"27.

Casos como el de Home Meal y Kodak demuestran la intención de compañías serias y establecidas, no sólo startups, de emplear las ICOs como la vía óptima de financiamiento. Esta intención se topa con una falta de regulación al que acogerse que le brinde al inversionista la debida seguridad y confianza.

En una revisión de la literatura sobre el tema regulatorio de las criptomonedas y las ICOs encontramos que las últimas investigaciones dan cuenta de los vacíos regulatorios y oportunidades de mejora, en materia de, por ejemplo, la protección contra la falsificación y seguridad (McKinney, Shao, Rosenlieb, \& Shao, 2015); las monedas virtuales como herramienta para delitos informáticos en el entorno virtual (lavado de dinero, financiación del terrorismo, etc.) (Atli, 2016); regulación y resolución de disputas (McKinney, Shao, Rosenlieb, \& Shao, 2015); ubicación física de unidades de criptomoneda para fines jurisdiccionales, características técnicas y su interacción con las leyes (McReynolds, Lerner, Scott, Roesner, \& Kohno, 2015); elaboración de la regulación con gestión de stakeholders (Tu \& Meredith, 2015); inconvenientes de las ICOs: falta de reglamentación, riesgos considerables, falta de responsabilidad--más del 10\% de las ICO a nivel mundial han resultado en estafas sustanciales, phishing, esquemas Ponzi y otros engaños (Chohan, 2017).

\section{CONCLUSIONES}

Con base en los hechos, casos y revisión de la literatura presentados en esta investigación, se llega a las siguientes conclusiones como respuesta a nuestra pregunta inicial: en cuanto a las ventajas potenciales y oportunidades de mejora que plantea el uso de las criptomonedas en crowdfunding y como herramienta para estrategias de crecimiento empresarial.

En cuanto a las ventajas en el uso de criptomonedas como estrategia de financiamiento, se identifican las siguientes:

1. La ICO es una estrategia que brinda cobertura y difusión a nivel mundial para la participación en la venta de una criptomoneda como medio de recaudación de capital.

2. La ICO es un medio más económico, porque permite evitar la intermediación de la banca tradicional.

27. Ver https://www.kippel01.com/pro/la-cnmv-tiende-una-manoa-nostrum-para-el-lanzamiento-de-su-ico.html 
3. Las criptomonedas, empleadas como título valor, permiten a sus tenedores participar del valor del crecimiento de la empresa, al subir el precio de las criptomonedas en el mercado secundario de intercambio.

4. Genera una "economía propia" alrededor de la empresa, reduciendo la necesidad de liquidez de moneda tradicional.

5. Como se evidenció en el caso de Crypterium, es posible emplear criptomonedas como herramienta combinada de financiamiento y de fidelización de clientes. Acordémonos que en esta estrategia se publicita los beneficios que se otorgan sobre los servicios o productos futuros a quienes las compren.
Los compradores estarían pre-pagando servicios o productos que adquirirán en el futuro. Esto permite ampliar la base de clientes y a su vez fidelizarlos pues a los tenedores de criptomoneda les resulta más beneficioso gastar los tokens dentro del sistema que cambiarlas para usarlas fuera, aunque tienen la opción de convertirlas nuevamente en divisas convencionales u otra criptomoneda en las casas de cambio virtuales.

En síntesis, y con el ánimo de hacer patente el efecto del uso de las estrategias de criptomonedas para potenciar el desarrollo de la empresa, se muestra a continuación la figura 2 en la que se puede ver una comparación, utilizando el lienzo de modelo de negocios ${ }^{28}$, de las situaciones tradicional y modificada:

Figura 2: Modelo de negocio común e incorporando la estrategia de criptomonedas (esquema superior: sin la estrategia; esquema inferior: con la estrategia). Elaborado empleando Canvas Business Model

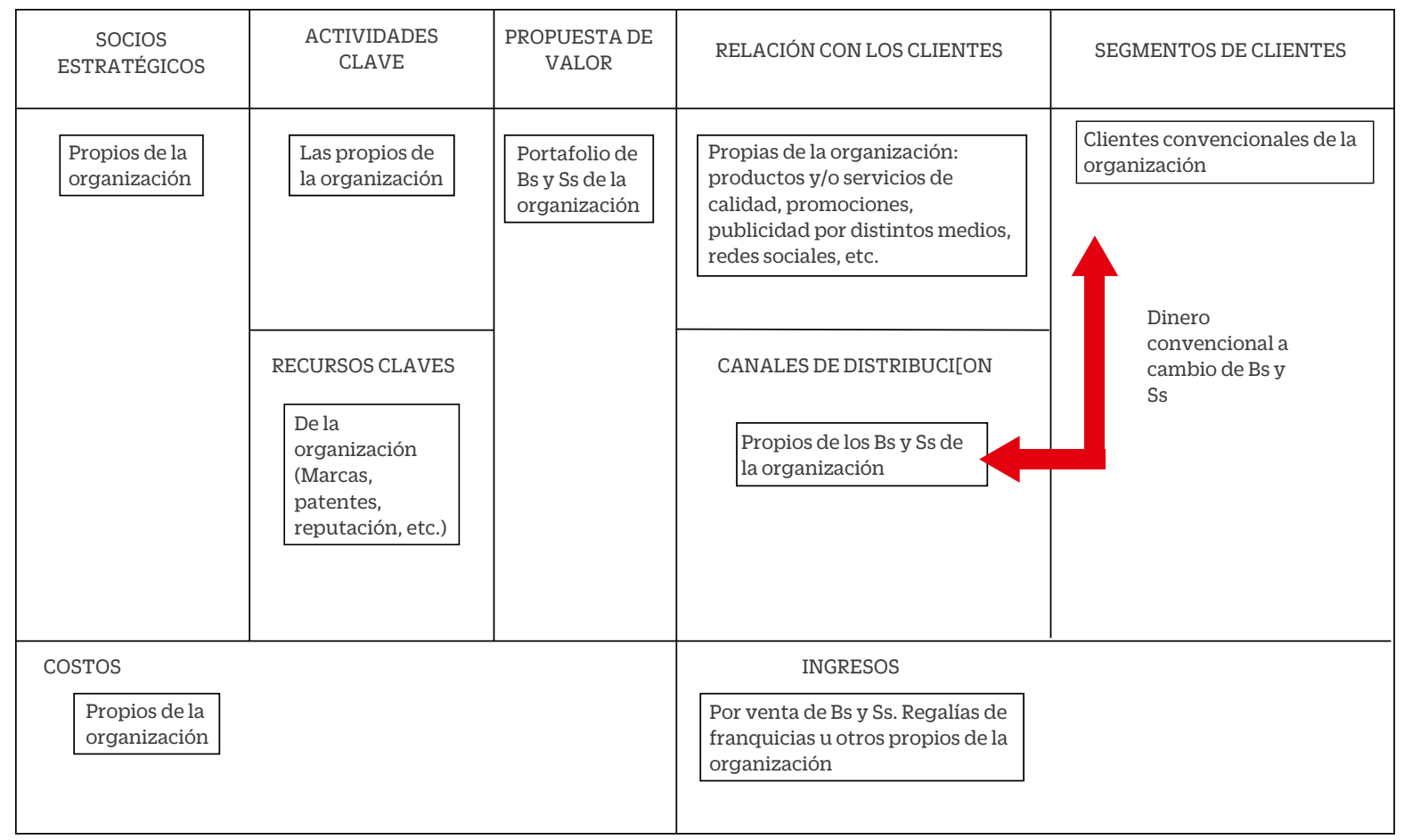




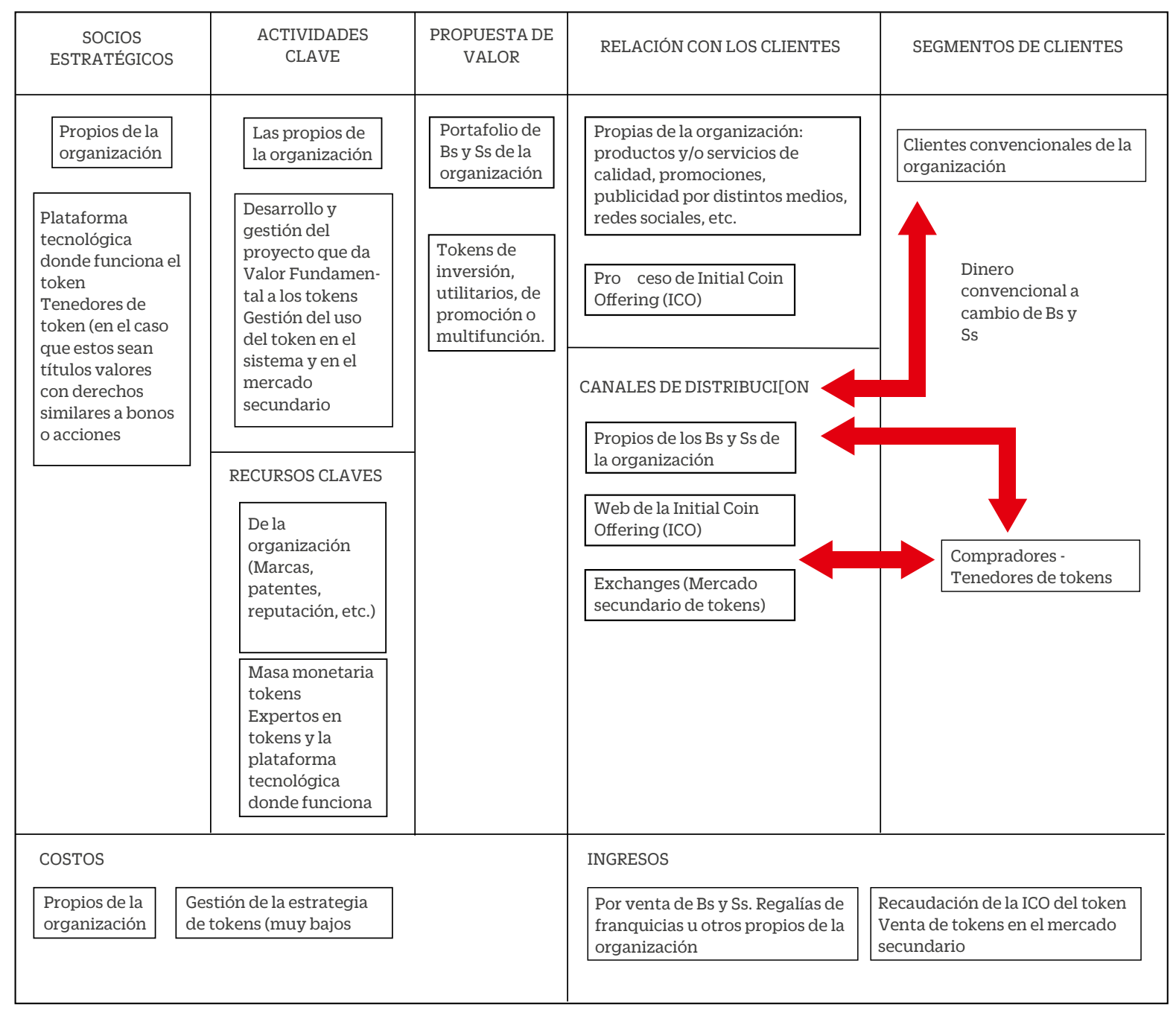

(https://strategyzer.com/canvas/business-model-canvas)

En cuanto a los factores de éxito en uso de criptomonedas como estrategia de funcionamiento, podemos concluir lo siguiente:

1. Se debe realizar la publicación de un White Paper donde se expliquen en detalle los aspectos técnicos del proyecto que está asociado a la criptomoneda, los aspectos de gestión relacionados con la emisión y los derechos que otorga la criptomoneda a los compradores-inversionistas. También debe comunicarse con transparencia en el documento, quién es el equipo central de personas responsables y quiénes asesoran o patrocinan de alguna forma el proyecto.

2. Se requiere diseñar una estrategia de emisión y preventa para atraer a los inversionistas, que pase por generar confianza buscando el aval o reconocimiento de instituciones reguladoras, en la medida que las leyes lo permitan.

3. Se requiere también, una estrategia de manejo de la masa monetaria para el control del precio en el mercado secundario especulativo.
4. Es necesario otorgar a los tenedores de las criptomonedas suficientes beneficios de uso de las mismas y comunicarlas adecuadamente para garantizar el interés y acogida del público comprador.

Finalmente, en cuanto a las oportunidades de mejora respecto al uso de criptomonedas como estrategia de funcionamiento, se ve que en el marco regulatorio resulta necesario trabajar, entre otros frentes, en los siguientes:

1. Exigir a las empresas emisoras la recaudación del dinero a través de un fideicomiso y el establecimiento de una autoridad o auditor externo que apruebe la liberación de los fondos contra el avance del proyecto.

2. Exigir el registro, ante la autoridad reguladora, de la identidad de las personas que hagan una compra mayor a un monto significativo; esto, como un disuasivo para el empleo de la compra mayor a un monto significativo; esto como un disuasivo para el empleo de la compra como un instrumento de lavado de dinero.

3. La exigencia, por parte del ente regulador, de la acreditación de un equipo calificado que respalde el proyecto y el manejo de la emisión y gestión de la criptomoneda. 
4. La exigencia, por parte del ente regulador, de que la empresa emisora y su proyecto sean clasificados por dos instituciones clasificadoras de riesgo y que dicha clasificación sea comunicada en la web de difusión de la ICO.

5. Regulación y auditoría de las empresas que desempeñan la función de casas de cambio virtuales.

6. La educación y advertencia a los compradores de que la inversión en criptomonedas en empresas nuevas es inversión de riesgo.

El uso de las criptomonedas sigue desarrollándose. Es muy probable que bitcoin y las principales criptomonedas de uso general logren establecerse en el mercado y evolucionen institucionalmente con el tiempo. Lo más seguro es que, en menos de 10 años, formen parte de nuestra vida cotidiana y podamos adquirir con ellas una gran variedad de bienes y servicios.

Respecto a su aplicación como estrategia de crecimiento empresarial, en un futuro se podrá ver a muchas empresas creando sus propias criptomonedas para su empleo en diversas formas con el fin de potenciar su crecimiento; empleándolas como medio de financiamiento a modo de bonos o acciones, sea a través de ICOs o de nuevas modalidades. Otras compañías las podrán emplear como "vales" que podrán canjearse a futuro por servicios u otros beneficios, en un contexto de un programa de fidelización, por ejemplo.

La creatividad siempre marca la pauta en el mundo empresarial. Solo es necesario que los gobiernos apoyen estas nuevas iniciativas creando la legislación mínima necesaria para garantizar la seguridad y la confianza a los usuarios.

\section{REFERENCIAS}

Atli, D. (2016). Cybercrimes via virtual currencies in international business. En M. Moore (Ed.), Cybersecurity Breaches and Issues Surrounding Online Threat Protection (pp. 121-143). doi:10.4018/978-1-52251941-6.ch006

Chohan, U. W. (2017, noviembre). Initial Coin Offerings (ICOs): Risks, Regulation, and Accountability. (Discussion Paper Series: Notes on the 21st Century). doi:10.2139/ssrn.3080098

De Albuquerque, B. S., \& De Castro Callado, M. (2015). Understanding Bitcoins: Facts and Questions. Revista Brasileira de Economia, 69(1), 3-16. doi:10.5935/00347140.20150001

Revista Brasileira de Economia, 69(1), 3-16. doi:10.5935/0034 7140.20150001

McKinney, R. E., Shao, L. P., Rosenlieb, D. C., \& Shao, D. H. (2015). Counterfeiting in cryptocurrency: An emerging problem. En D. L. Kuo Chuen (Ed.), Handbook of Digital Currency. Bitcoin, Innovation, Financial Instruments, and Big Data (pp. 173-187). doi: 10.1016/B978-0-12802117-0.00008-4

McReynolds, E., Lerner, A., Scott, W., Roesner, F., \& Kohno, T. (2015). Cryptographic currencies from a tech-policy perspective: Policy issues and technical directions. En M. Brenner, N. Christin, B. Johnson \& K. Rohloff (Eds.), Lecture Notes in Computer Science Series: Vol. 8976. FC 2015. Financial Cryptography and Data Security (pp. 94-111). doi:10.1007/978-3-662-48051-9_8

Nakamoto, S. (2008). Bitcoin: A Peer-to-Peer Electronic Cash System. Obtenido en https://bitcoin.org/bitcoin.pdf

Tu, K. V., \& Meredith, M. W. (2015). Rethinking virtual currency regulation in the bitcoin age. Washington Law Review, 90(1), 271-347. Obtenido en https:// www.questia.com/library/journal/1P3-3677988841/ rethinking-virtual-currency-regulation-in-the-bitcoin 